\title{
La qualité des services : une recherche sur le prêt entre bibliothèques dans les grandes bibliothèques publiques du \\ Canada
}

\section{Interlibrary Loan Services in Large Canadian Public Libraries:} The Quality of Services

\section{La calidad de los servicios: una investigación sobre el préstamo entrebibliotecas en las grandes bibliotecas públicas del Canadá}

\section{Françoise Hébert}

Volume 41, numéro 4, octobre-décembre 1995

URI : https://id.erudit.org/iderudit/1033204ar

DOI : https://doi.org/10.7202/1033204ar

\section{Aller au sommaire du numéro}

\section{Éditeur(s)}

Association pour l'avancement des sciences et des techniques de la documentation (ASTED)

\section{ISSN}

0315-2340 (imprimé)

2291-8949 (numérique)

Découvrir la revue

\section{Citer cet article}

Hébert, F. (1995). La qualité des services : une recherche sur le prêt entre bibliothèques dans les grandes bibliothèques publiques du Canada.

Documentation et bibliothèques, 41(4), 217-224.

https://doi.org/10.7202/1033204ar

\section{Résumé de l'article}

Une recherche sur le prêt entre bibliothèques dans les 38 plus importantes bibliothèques publiques au Canada illustre ce qui s'est passé quand les 130 participants sont entrés dans leur bibliothèque à la recherche d'un livre que la bibliothèque ne possédait pas. Leur démarche a obtenu un taux de succès de $52 \%$ dans un délai moyen de 38 jours, résultats décevants du point de vue des bibliothèques. Du point de vue des usagers, une comparaison de leurs attentes et de leurs perceptions a révélé que la plupart ont été déçus du service obtenu. Les gestionnaires de bibliothèque se doivent d'améliorer l'efficacité du service par des moyens qui vont aussi améliorer la satisfaction des usagers.
Tous droits réservés (C) Association pour l'avancement des sciences et des techniques de la documentation (ASTED), 1995
Ce document est protégé par la loi sur le droit d'auteur. L’utilisation des services d’Érudit (y compris la reproduction) est assujettie à sa politique d'utilisation que vous pouvez consulter en ligne.

https://apropos.erudit.org/fr/usagers/politique-dutilisation/ 


\title{
La qualité des services: une recherche sur le prêt entre bibliothèques dans les grandes bibliothèques publiques du Canada
}

\author{
Françoise Hébert \\ Consultante en ressources de l'information, Toronto
}

Une recherche surle prêt entre bibliothèques dans les 38 plus importantes bibliothèques publiques au Canada illustre ce qui s'est passé quand les 130 participants sont entrés dans leurbibliothèque à la recherche d'un livre que la bibliothèque ne possédait pas. Leur démarche a obtenu un taux de succès de $52 \%$ dans un délai moyen de 38 jours, résultats décevants du point de vue des bibliothèques. Du point de vue des usagers, une comparaison de leurs attentes et de leurs perceptions a révélé que la plupart ont été déçus du service obtenu. Les gestionnaires de bibliothèque se doivent d'améliorer l'efficacité du senvice par des moyens qui vont aussi améliorer la satisfaction des usagers.

\section{Interlibrary Loan Services in Large Canadian Public Libraries: The Quality of Services}

An unobtrusive study of interlibrary loan in Canada's 38 largest public libraries documented what happened when 130 participants asked for a book that the library did not own. The usual indicators of quality used in libraries showed a fill rate of $52 \%$ and a tumaround time of 38 days; poor results by any standard. Customer measures of quality, based on a comparison of expectations and perceptions, showed that most participants were disappointed with the service that they received. Library management must improve service in ways that will also improve customer satisfaction.
La calidad de los servicios: una investigación sobreel préstamo entrebibliotecas en las grandes bibliotecas públicas del Canadá

Una investigación sobre el préstamo entrebibliotecas en las 38 más grandes bibliotecas públicas en Canadá ilustra los resultados obtenidos cuando 130 participantes han consultado su biblioteca para obtener un libro que la biblioteca no poseía. Solamente $52 \%$ de los libros fueron obtenidos en un periodo promedio de 38 dias. Del punto de vista de los usuarios, una comparación entre sus expectativas y sus percepciones ha revelado que la mayoria se sentia frustrada por aquel servicio obtenido. Los administradores de las bibliotecas deben mejorar la eficiencia del servicio por medios que van también a mejorar la satisfacción de los usuarios.
Dans le domaine des bibliothèques, pour mesurer la qualité de nos services, nous avons tendance à mettre l'emphase sur les intrants et les extrants.

- Les intrants comprennententre autres les budgets, le personnel et les collections. Ces données sont faciles à mesurer et nous le faisons fréquemment. Nous publions les mesures dans nos rapports annuels et les utilisons à des fins de coopération. Ces données, cependant, mesurent seulement le ressources de la bibliothèque pour donner des services; elles ne sont pas utiles pour évaluer les services comme tels.

- Les extrants représentent les services actuellement fournis par la bibliothèque, par exemple le nombre de livres prêtés et le nombre de questions de référence reçues. II est facile de quantifier les extrants, mais il est moins facile d'en évaluer la qualité. Par exemple, on peut compter le nombre de questions de référence, mais il est plus difficile de juger si les réponses données aux questions étaient satisfaisantes. On peut compter le nombre de personnes qui entrent dans la bibliothèque, mais il demeure difficile d'établir si ces personnes ont obtenu ce qu'elles cherchaient et, le cas échéant, plus difficile encore de déterminer pourquoi elles ne l'ont pas obtenu, en somme des informations qui permettraient de combler les lacunes.

Une mesure appropriée des extrants devrait indiquer non seulement la quantité de travail effectué, mais aussi la façon dont il est accompli, c'est-à-dire la qualité du service. Les meilleures mesures de qualité tiendront compte à la fois de la perspective de la bibliothèque et de celle de l'usager.
Dans les bibliothèques, presque toutes les mesures que nous utilisons pour déterminer la qualité de nos services, ont été développées uniquement du point de vue organisationnel, tout en ignorant la perspective de l'usager. Le marketing de service propose des concepts qui incitent à réfléchir sur les services offerts dans nos bibliothèques, et même à s'interroger sur la valeur des mesures utilisées jusqu'à maintenant pour mesurer leur qualité.

\section{Le concept de service}

Un service se définit difficilement. II est à la fois un effort, une action, une performance, une expérience, une activité ayant une valeur pour un client, un résultat. Ces définitions sont incomplètes, et les chercheurs ont davantage réussi à définir les services en énumérant les attributs quiles distinguent des produits. Quels sont ces attributs? 


\section{L'intangibilité}

D'abord, les services sont intangibles. Leur objectif est de créer une expérience pour quelqu'un (Shostack 1985 , 245). Un service ne s'étale pas dans une vitrine comme un produit. Souvent, les gens ne savent pas à quoi s'attendre quand ils font appel à un service. Les usagers vont à la bibliothèque pour trouver une réponse à une question, ils espèrent trouver ce qu'ils cherchent, mais ils n'ont aucune garantie de succès.

Les premiers contacts entre la bibliothèque et l'usager sont extrêmement importants parce que les usagers ne savent pas à quoi s'attendre en faisant appel à un service surtout la première fois (Booms and Nyquist 1981, 173). La première interaction affecte de façon critique la perception de la qualité.

Beaucoup d'usagers peuvent difficilement différencier un bon service d'un mauvais. II leur est difficile de juger si la collection de la bibliothèque est bien développée, si le catalogue est conforme aux normes, si le personnel est compétent. Les usagers sont confiants et décident de la qualité du service après coup. Leur jugement est subjectif plutôt qu'objectif, fondé davantage sur l'ambiance de la bibliothèque que sur la compétence professionnelle des employés. Lorsqu'il s'agit de mesurer la qualité des services de bibliothèque, quelle perspective a davantage d'importance: ce que l'usager pense de la bibliothèque ou la performance de la bibliothèque? Dans le meilleur des mondes, les perspectives de l'usager et de l'institution coïncident. Cependant, cela est difficile à déterminer.

\section{L'interaction entre personnel et usa- gers}

Un deuxième attribut des services est qu'ils requièrent la participation de l'usager. Les employés et les usagers travaillent ensemble pour atteindre un but, et le comportement de l'usager est aussi important que celui du personnel. La direction doit former les usagers pour que leur contribution au service soit utile et contrôler leur comportement afin d'assurer la qualité du service (Bowen and Cummings 1990, 5). Ce n'est pas une mince tâche. La direction doit s'assurer que l'aménagement spatial de la bibliothèque est adéquat, la signalisation efficace, le matériel à sa place sur les rayons, le service de renseignements visible, l'ambiance agréable, le personnel disponible et sympathique. Des études effectuées dans les bibliothèques publiques et universitaires ont démontré que les usagers préfèrent une bibliothèque avec libre-service. Nous avons l'obligation de former nos usagers à bien utiliser les bibliothèques avec libre-service et de nous assurer qu'il leur est facile d'obtenir de l'aide quand ils en ont besoin.

II faut aussi former nos usagers à avoir des attentes réalistes. L'usager qui commande un livre par le truchement du prêt entre bibliothèques et qui s'attend à le recevoir dans un délai de trois heures, a une attente irréaliste. Les usagers doivent apprendre ce que la bibliothèque peut faire pour eux et ce qu'elle ne peut pas faire. Comment procéder? À ce jour, les bibliothèques n'ont pas connu un énorme succès en ce qui concerne la formation de leurs usagers.

\section{La simultanéité de la production et de} la consommation du service

Un troisième attribut des services est qu'ils sont produits et consommés simultanément. La qualité se détermine lors de l'interaction entre le personnel et l'usager. Le préposé au service joue un role clé dans le processus de détermination de la qualité (Bowen and Cummings 1990, 5). La qualité du service peut varier d'un employé à l'autre, d'une journée à l'autre, même d'une heure à l'autre. Le contrôle de la qualité est difficile et il est impossible de savoir avec certitude si les services fournis par le préposé correspondent aux attentes de la direction (Bitner and Zeithaml 1987, 8). Vous allez à la bibliothèque et voilà que la personne au service de l'information jase avec un copain pendant que vous attendez. Même si vous n'attendez que vingt secondes, vous en voulez à la personne et à la direction de la bibliothèque. Peu importe la qualité du service que vous aurez finalement reçu, vous demeurez mécontent. Votre mécontentementn'a rien à voir avec la qualité objective du service reçu et tout à voir avec la qualité subjective de la façon dont le service est rendu. La gestion de l'interaction entre l'employé et l'usager ne se limite pas à entraîner le personnel à sourire poliment à l'usager. Une gestion efficace exige d'abord de comprendre les comportements qui produisent une impression de bon service et, par la suite, de former et motiver le personnel à se comporter de cette façon.

Un aspect important de la gestion de l'interaction est la manière dont le personnel réagit lors d'un échec de service, par exemple lorsque le numéro d'une revue ne se trouve pas à sa place habituelle, ou encore lorsque la photocopieuse tombe en panne. La volonté et l'habileté du personnel à faire face à de tels problèmes peuvent grandement influencer l'attitude de l'usager envers le service. Lorsque la photocopieuse dans une bibliothèque tombe en panne par exemple, le personnel peut s'excuser auprès de l'usager et l'aider à trouver une autre photocopieuse. Un geste plus positif encore serait d'offrir l'appareil réservé au personnel pour que l'usager puisse terminer ses photocopies sans frais. Voilà un usager fort satisfait, malgré l'échec du service. Les chercheurs ont découvert que fréquemment une explication arrivera à satisfaire l'usager lors d'un problème de service (Parasuraman 1987, 23). On peut quelquefois avoir recours à une explication toute faite, mais souvent le personnel doit élaborer une réponse adaptée à la situation. Le personnel doit donc comprendre le fonctionnement de la bibliothèque et le genre d'explications qui pourraient satisfaire l'usager.

\section{Théorie de l'attribution}

Selon la théorie de l'attribution, lorsqu'il y a un échec de service, le client, même inconsciemment, cherche à qui la faute (Folkes 1984, 407). Si la photocopieuse ne fonctionne pas à cause d'une panne d'electricité causée par une tempête de neige, les usagers ne blâmeront pas la bibliothèque. Mais si la photocopieuse ne fonctionne pas parce que quelqu'un a oublié de commander du papier, les usa'gers vont raisonnablement blâmer la bibliothèque.

Le personnel doit apprendre à fournir des explications qui vont minimiser les risques de blâmer la bibliothèque lors d'un échec de service. Les compagnies aériennes ont bien compris la théorie de l'attribution: Lorsque vous attendez impatiemment sur la piste de décollage, le capitaine 
expliquera tout de suite ce qui se passe s'il ne s'agit pas d'un problème causé par la compagnie. II vous dira rapidement que la tour de contrôle à Toronto cause votre retard à Montréal, mais il ne vous dira jamais que le délai a été causé par un mécanicien arrivé ivre au travail.

\section{Le concept de qualité}

Lorsqu'il s'agit de produits comme des automobiles ou des ordinateurs, la qualité se mesure par la conformité aux normes (Klaus 1985, 19). Une compagnie décide ce qu'elle veut construire, les ingénieurs s'occupent du design et des spécifications, et les travailleurs fabriquent le produit suivant les spécifications. La qualité se mesure d'après les attributs du produit. La plupart des mesures de qualité développées dans le secteur des bibliothèques suivent ce concept de qualité basé sur les attributs du service. L'État du Wisconsin aux États-Unis, par exemple, spécifie que pour les prêts entre bibliothèques dans le réseau public, $30 \%$ des demandes doivent être satisfaites dans un délai de sept jours et $70 \%$ dans un délai de trente jours (Davis et Daniel 1991, 131). Si les bibliothèques publiques du Wisconsin respectent ou surpassent ces normes, elles se félicitent de la qualité de leur service.

Cependant, le concept de qualité basé sur les attributs du service a été critiqué parce qu'il est objectiviste, statique et rationaliste (Klaus 1985, 22). II calcule la qualité uniquement du point de vue de l'institution et non du point de vue de l'usager. C'est une faiblesse importante. Un concept alternatif, développé afin de corriger cette faiblesse, propose une mesure de qualité basée sur la satisfaction du client (Klaus 1985, 21). Ce concept reconnaît que la décision de quelqu'un d'utiliser un service une première fois, d'y faire appel de nouveau, ou de le recommander à d'autres, est basée sur une évaluation subjective de l'interaction de service et non sur la conformitè du service à une norme institutionnelle. La qualitè se détermine lorsque les usagers comparent ce qu'ils attendent d'un service avec ce qu'ils obtiennent de ce service ou en d'autres termes lorsqu'ils comparent leurs attentes à leurs perceptions.

\section{Attentes et perceptions}

Les attentes sont le résultat d'une combinaison de facteurs: ce que les autres ont déjà dit du service, les expériences personnelles avec l'institution ou avec d'autres institutions semblables. Chaque usager arrive à la bibliothèque avec certaines attentes. Elles sont individuelles et la bibliothèque ne peut les connaître à l'avance.

Les perceptions sont le fruit de l'évaluation qu'une personne fait après avoir reçu un service. Le processus d'évaluation comporte des éléments techniques et fonctionnels, selon le modèle développé par Grönroos (1984, 38-39).

Tableau 1: Le modèle de qualité Grönroos (1984)

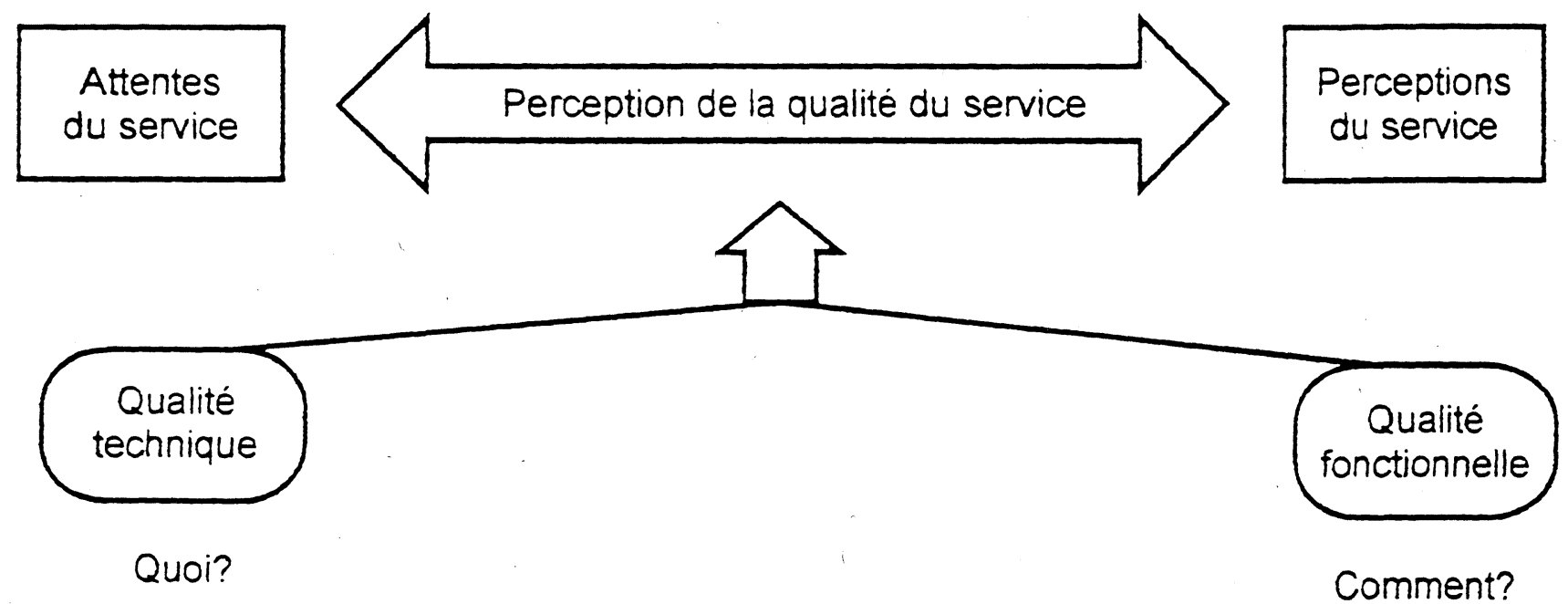


L'élément technique du processus d'évaluation répond à la question "Que s'est il passé?» L'usager a-t-il trouvé le livre qu'il cherchait? A-t-il reçu la réponse exacte à sa question? La perspective subjective de l'usager n'est pas nécessairement la même que la perspective objective de la bibliothèque. Par exemple, dans le cas du prêt entre bibliothèques, l'usager va évaluer la qualité technique du service en fonction de son besoin individuel et non en fonction d'une norme qui dicte que la bibliothèque a bien réussi lorsque $70 \%$ des transactions sont complétées dans un délai de trente jours. L'élément fonctionnel du processus d'évaluation répond à la question "Comment me suis-je senti?» II s'agit encore une fois d'une mesure subjective qui indique comment l'usager a perçu le service lors de son interaction avec la bibliothèque.

Le processus d'évaluation qui détermine la perception de l'usager dépend donc de ce qui s'est passé et de comment cela s'est passé. L'usager compare ses perceptions avec ses attentes. Si le service qu'il a reçu dépasse ses attentes, le résultat de l'interaction est positif et l'usager est satisfait. Si le service n'a pas répondu à ses attentes, le résultat est négatif et l'usager est mécontent (Oliver and DeSarbo 1988, 495).

Imaginons par exemple notre usager qui arrive à la bibliothèque, va directement au service de renseignements et pose la question «Avez-vous ce livre?» Le bibliothécaire lui indique le catalogue et répond avec impatience: «C'est indiqué partout qu'il faut consulter le catalogue d'abord. Revenez si vous nous le trouvez pas là.» Humilié, notre pauvre usager file vers le catalogue, fouille pour la cote et trouve le livre exactement là où il devait être sur les rayons. Du point de vue de la bibliothèque, le livre désiré a été trouvé et le service est un succès. Mais il est probable que l'usager est mécontent de la qualité du service qu'il a reçu. II faut se rappeler que les perspectives de l'usager et de la bibliothèque ne sont pas toujours semblables. Un service réussi peut créer un usager mécontent tandis qu'un échec de service peut quelquefois créer un usager satisfait. Le "comment» du service est aussi important que le «quoi» (tableau 1).

\section{Un projet de recherche}

Le concept de qualité de service basé sur une comparaison des perceptions et des attentes de l'usager s'applique à la gamme entière de services offerts dans les bibliothèques. Mais comment évaluer la qualité d'un service du point de vue de l'usager? Un projet de recherche s'est penché sur cette question en examinant un service spécifique: le prêt entre bibliothèques.

Du point de vue de la bibliothèque, les critères usuels pour mesurer la qualité du prêt entre bibliothèques sont le taux de succès et les délais requis (Lancaster 1988, 140; Waldhart 1985, 317; Van House et al 1987,62). Du point de vue de l'usager, un instrument développé par des chercheurs américains, SERVQUAL, a permis de mesurer les attentes et les perceptions (Parasuraman, Zeithaml and Berry 1988). Le but de la recherche a été, pour les mêmes transactions, de comparer les mesures de qualité utilisées par les bibliothèques et les mesures de qualité utilisées par les usagers pour déterminer si elles étaient congruentes.

\section{Méthodologie}

Quatre personnes ont été recrutées dans chacune des 38 plus grandes villes du Canada. Ces 152 participants ont d'abord complété le questionnaire SERVQUAL afin de mesurer leurs attentes générales des services dans les bibliothèques publiques. Les participants ont ensuite reçu des instructions détaillées sur un rôle à jouer dans leur bibliothèque locale. En premier lieu, ils iraient à la bibliothèque, avec en main la photocopie d'une page contenant une référence à un livre spécifique. Si le livre ne se trouvait pas dans le catalogue, les participants se dirigeraient ensuite vers le service de renseignements pour dire à la personne de service, "Excusez-moi... J'ai besoin de ce livre mais je ne le trouve pas dans votre catalogue. Pouvez-vous m'aider?»

Les livres en question avaient été contrôlés préalablement par le chercheur. Quatre livres inventés pour l'occasion ont été fabriqués en 38 exemplaires chacun.
La Bibliothèque nationale du Canada a mis les livres $A$ et $B$ sur ses rayons, bien catalogués et ajoutés à la base de données $D O B I S$ indiquant la Bibliothèque nationale du Canada comme source unique. La bibliothèque de l'Université de Toronto en a fait autant pour les livres $C$ et $D$, qui ont été ajoutés à la base de données CATTS de ISM. Les bibliothèques cherchant ces livres les trouveraient facilement en vérifiant dans DOBIS ou CATTS ou encore en faisant appel au service de localisation de la Bibliothèque nationale du Canada. Autrement dit, ces livres étaient faciles à trouver.

Les 152 participants ont tous tenu un journal pour consigner leurs démarches au cours de l'exercice. À la fin, ils ont complété la deuxième partie du questionnaire SERVQUAL ce qui a permis de mesurer leurs perceptions du service reçu. SERVQUAL a donc fourni les données sur les attentes et les perceptions des usagers. Le journal des participants et les dossiers de la Bibliothèque nationale du Canada et de l'Université de Toronto ont fourni les données qui ont permis de vérifier le taux de succès et les délais requis pour les 130 transactions qui ont été documentées jusqu'à la fin de l'exercice.

Résultats: perspective institutionnelle

Le projet a obtenu un taux de succès de $52 \%$. Sur un total de 130 demandes, les bibliothèques publiques ont carrément refusé 14 demandes; elles n'ont pas réussi à localiser le livre 41 fois; elles ont refusé de procéder à trois demandes après avoir localisé le livre dans une bibliothèque d'université et quatre fois, elles n'ont pas pu trouver le livre emprunté quand le participant est venu le chercher à la bibliothèque.

Les délais requis pour compléter les transactions ont varié de 4 à 130 jours, avec une médiane de 38 jours. En moyenne, les bibliothèques ont laissé passer 23 jours avant d'essayer de localiser les livres demandés dans DOBIS ou CATTS ou bien en communicant avec le service de localisation de la Bibliothèque nationale du Canada. Rappelons qu'il s'agit des 38 plus importantes bibliothèques du Canada et 


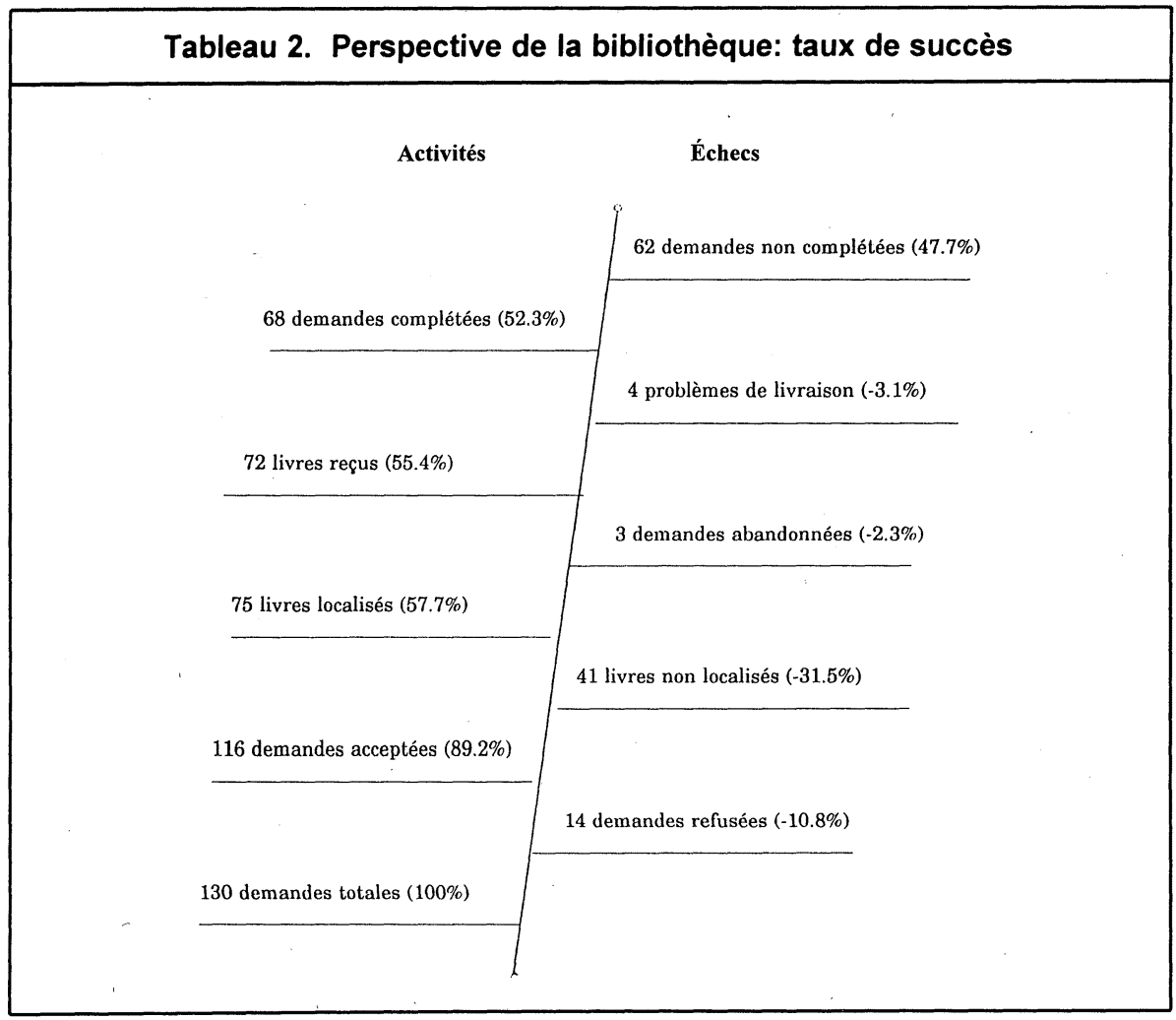

que les livres demandés étaient faciles à trouver. Du point de vue des bibliothèques, ces résultats sont décevants.

\section{Résultats: les attentes et perceptions des usagers}

Les scores du questionnaire SERVQUAL mesurant les attentes des participants sur la qualité des services dans une "bonne» bibliothèque étaient élevés, la moyenne étant de 5,6 sur 7 . Une analyse factorielle a révélé cinq facteurs selon lesquels les participants ont évalué la qualité des services:

Fiabilité L'habileté de rendre un bon service de façon précise et fiable

Capacité La volonté d'aider l'usager de répondre et de rendre le service avec célérité

Confiance La compétence et les connaissances du personnel

Bienveillance L'attention individuelle et chaleureuse que le personnel offre aux usagers

Les participants ont classé ces facteurs par ordre d'importance: la fiabilité du service étant considérée comme le plus important et la tangibilité des services comme le moins important (tableau 3).

Les scores du questionnaire SERVQUAL mesurant les perceptions de la qualité du service de prêt ont été plus bas, soit 5,2 sur 7 . Les perceptions ne correspondant pas aux attentes: le résultat est négatif, c'est-à-dire que la plupart des participants ont été déçus du service obtenu. Plus la différence entre les perceptions et les attentes est négative, plus le manque de qualité est grave. Dans cette recherche, le facteur le plus important, la fiabilité, a reçu le score le plus négatif. Le deuxième facteur le plus important, la capacité de répondre à la demande, a reçu le deuxième score le plus négatif. Le facteur le moins important a reçu le seul score positif. II y a donc eu un écart important entre les attentes des usagers et leur perception de la qualité du service qu'ils ont obtenu dans cette recherche (tableau 4).

La corrélation a été très faible (.173

Tangibilité L'environnement physique, l'équipement, les publications, l'ambiance de la bibliothèque

Tableau 3. Importance relative des facteurs de qualité

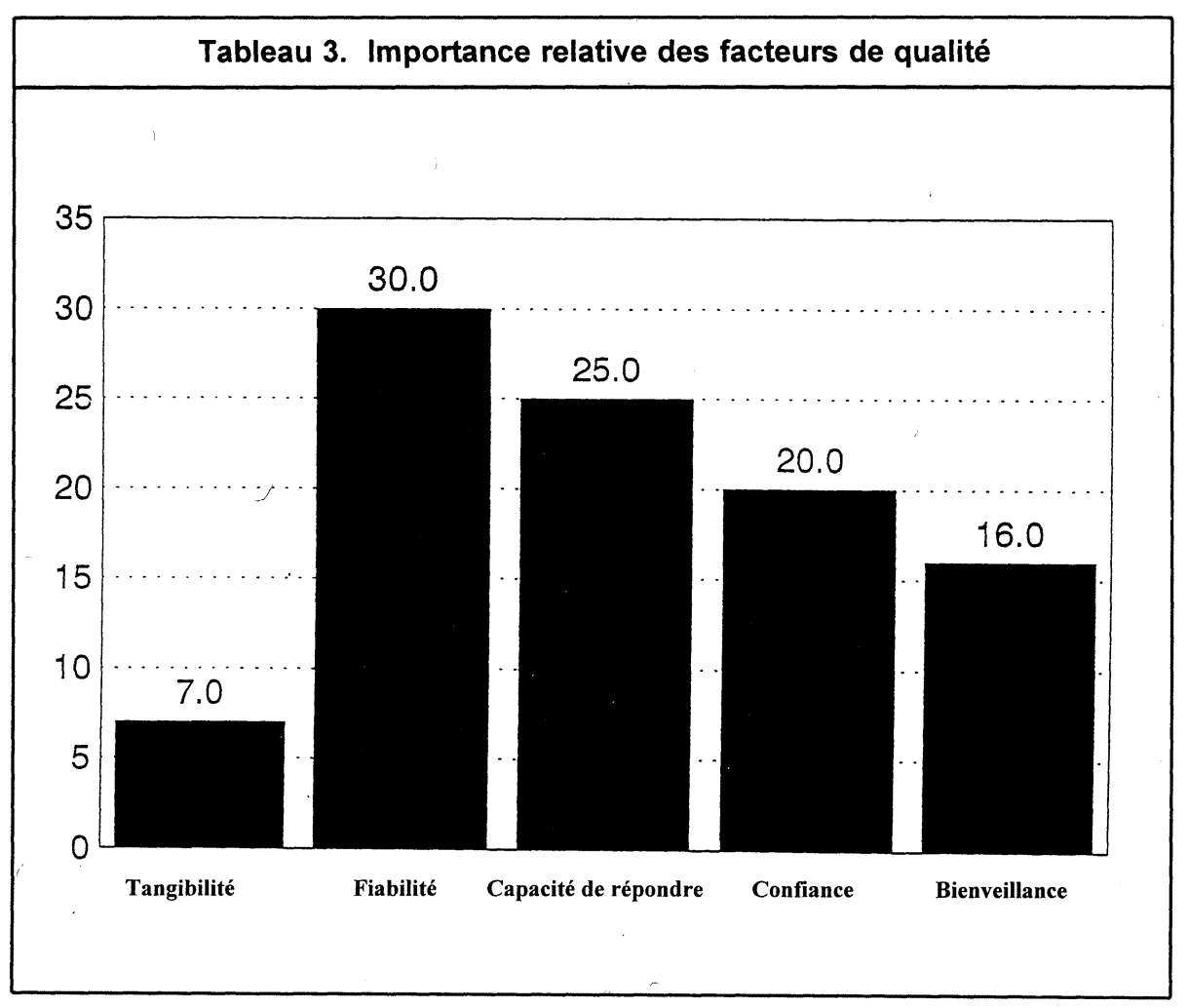




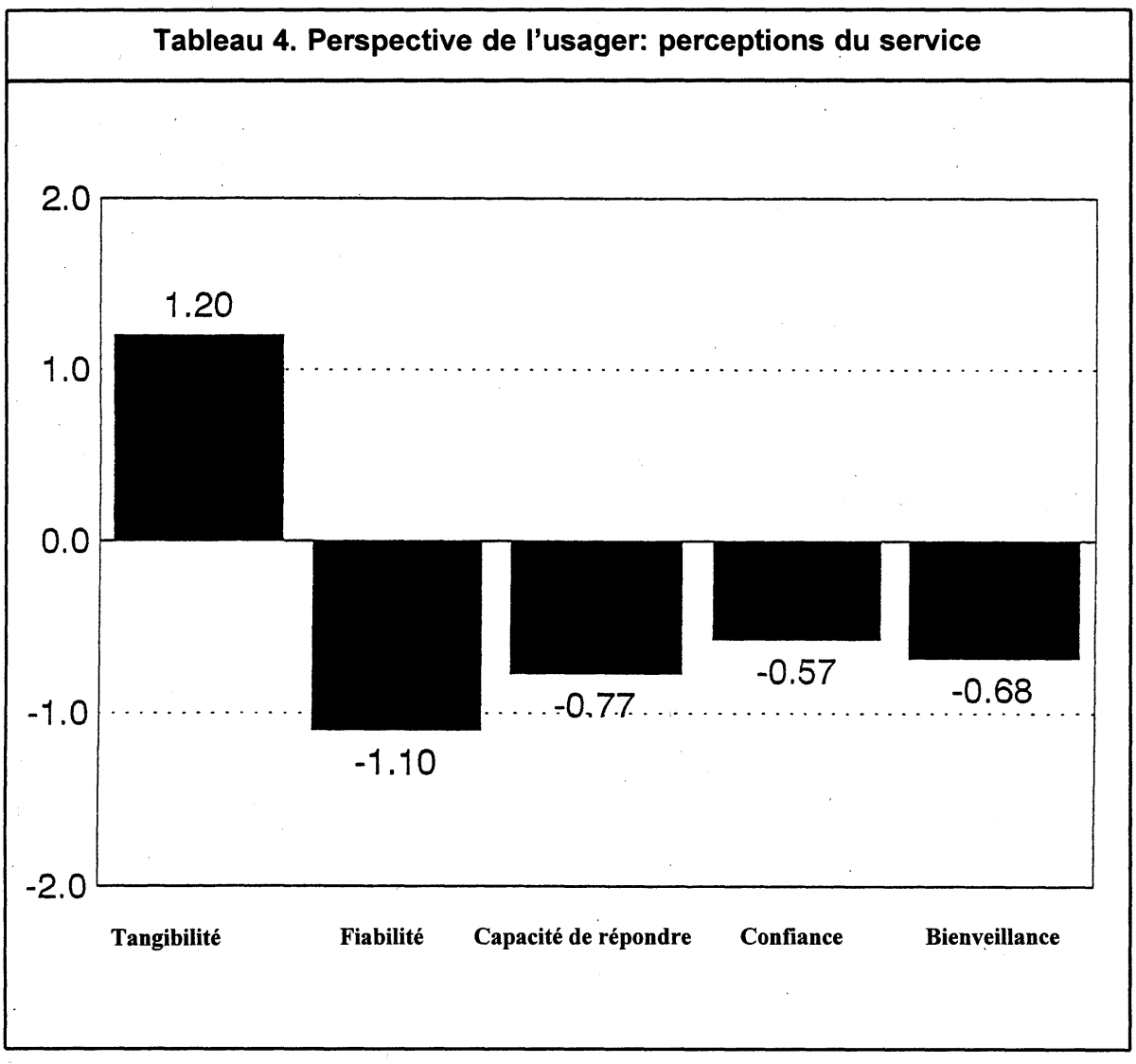

(comparaison des attentes et des perceptions). Ceci indique qu'un certain nombre des participants qui ont reçu un bon service du point de vue de la bibliothèque (ils ont reçu le livre rapidement) ont considéré avoir reçu un mauvais service et ont été déçus. Par contre, certains participants qui n'ont pas reçu le livre demandé se sont déclarés très satisfaits du service. Cela permet de confirmer que dans bien des cas, le "comment» d'un service est aussi important que le «quoi». Le comportement des employés au service de l'information peut déterminer la satisfaction de l'usager, peu importe si la bibliothèque a fourni le service demandé ou non. Améliorerl'efficacité d'un service, par exemple en réduisant les délais requis pour le prêt entre bibliothèques, ne fera pas plus de clients satisfaits si le personnel est incompétent ou impoli.

Laissons parler les participants à cette recherche. II faut remarquer combien souvent les participants notent les facteurs "comment» plutôt que les facteurs «quoi» dans leurs commentaires sur la qualité du service qu'ils ont reçu:
Livre trouvé / score excellent

La personne m'a expliqué exactement ce qu'elle ferait pour trouver ce livre. Elle m'a indiqué à quelle date elle m'appellerait et elle l'a fait.

Livre trouvé / score excellent

Lorsquej'ai fait ma demande, le personnel n'était pas certain de pouvoir trouver le livre. On m'a dit qu'on essaierait mais que cela pourrait prendre un certain temps. Sept semaines plus tard le livre est arrivé et on m'a téléphoné tout de suite pourm'en informer. Le lendemain j'ai aussi reçu une carte postale d'eux. Quand je me suis présenté, on s'est bien occupé de moi. J'ai été satisfait du personnel et du senvice audelà de mes attentes. Le personnel a été excellent.

\section{Livre trouvé / score excellent}

Le personnel était bien informé, poli et efficace. II a été très courtois et - écoutez ceci - non seulement je n'avais pas ma carte de bibliothèque avec moi, mais elle était expirée depuis plusieurs mois. PAS DEPROBLEME! Bien que le personnel ait été de toute évidence irrité que je veuille aller chercher le livre à la bibliothèque de mon quartier et non à la bibliothèque centrale, il a continué à me foumir un bon service. II m'a informé du délai et suggéré diverses façons d'obtenir le livre plus rapidement. II a été remarquablement poli lorsque j'ai réfusé ses conseils. II a trouvé le livre, l'a inscrit à mon nom, m'a dit combien de temps il me le réserverait, ne m'a pas demandé un sou et ne m'a jamais bâillé en pleine figure.

Livre trouvé / score très bien

Le personnel était poli et efficace. La personne a traité efficacement ma demande. Elle a enregistré rapidement l'information requise. J'ai été sensible à sa manière de traiterma demande parce que la bibliothèque était sur le point de fermer. Elle m'a même offert un sourire. Le service dans cette bibliothèque m'a bien plu.

Livre pas trouvé / score excellent Les employés de la bibliothèque ont accepté de faire une recherche à travers le Canada pour trouver ce livre lorsqu'ils ont constaté qu'il n'était pas disponible dans ma province. Je suis certain qu'ils auraient tout tenté pour obtenir le livre si j'en avais eu véritablement besoin.

Livre pas trouvé / score excellent La bibliothécaire a été très polie et gentille. Elle a fait de son mieux pour trouverle livre mais m'a expliqué que le livre n'était pas disponible au Canada.

Livre pas trouvé / score excellent J'estime que les employés de la bibliothèque ont fait de leur mieux pour moi. Ils m'ont demandé l'auteur et le titre de mon ouvrage, ont vérifié dans tous les catalogues de livres et m'ont dit qu'ils s'informeraient auprès d'autres bibliothèques. Lorsqu'ils m'ont téléphoné, ils m'ont dit qu'ils avaient vérifié partout mais qu'ilsn'avaient pas réussi à trouverle livre. Ils ne voulaient pas poursuivre leurs recherches. J'ai aimé le service des employés de cette bibliothèque.

Livre pas trouvé / score minable Ils ont été très courtois et attentifs jusqu'au moment où ils ont vérifié et se sont aperçus que le livre n'était pas dans leur système. Le service s'est arrêté là. Ils m'ont tout simplement dit qu'il faudrait que je le localise moi-même. 
Livre pas trouvé / score minable IIs ne voulaient pas effectuer une recherche à cause de quelque chose appelée une "vérification». Ils ne m'ont pas dit ce que c'était, mais m'ont tout simplement dit qu'ils ne pouvaient pas "vérifier» le livre et que conséquemment ils ne tenteraient pas de le trouver. Fin de la discussion. Cette expérience m'a déconcerté.

Livre pas trouvé / score le plus minable J'aiparlé à la dame au service de référence et lui ai expliqué que j'avais vraiment besoin de ce livre mais que je ne pouvais pas le trouver dans le catalogue en ligne. Elle m'a dit: "Si vous ne pouvez pas le trouver dans le catalogue en ligne, c'est que nous ne l'avons pas». Je lui ai demandé de vérifier elle-même, au cas où j'aurais fait une erreur en raison de ma méconnaissance du système. À contrecoeur, elle a verifié et confirmé que la mention du livre ne s'y trouvait pas. Je me suis informée du service de prêt entre bibliothèques. Elle m'a dit qu'un tel service n'existait pas dans les bibliothèques. Je lui ai dit qu'un ami avait obtenu un livre de cette manière. Elle m'a répondu que je me trompais. Croyant quej'aurais plus de chance avec quelqu'un d'autre, je suis revenue pendant la fin de semaine et ai tenté ma chance encore une fois au service de référence. Après avoir vérifié que le livre n'apparaissait pas dans le catalogue en ligne, la dame m'a suggéré un autre livre. Je lui ai dit que je voulais le livre demandé au départ. Elle voulait savoir pourquoi ce livre et pas un autre. Je lui ai dit que ce livre contenait une citation dont j'avais besoin. Elle m'a suggéré de commander le livre dans une librairie. Je lui ai dit que je ne voulais pas acheter tout un livre pour une seule citation. Je me suis informée du service de prêt entre bibliothèques. Elle m'a dit qu'il n'était offert qu'aux professionnels. J'ai répondu qu'un ami avait emprunté un livre par ce service et qu'il n'était pas un professionnel. Elle m'a dit que peut-être la bibliothèque centrale pourrait le faire. Je lui ai demandé d'établir le contact pour moi étant donné que j'étais mère célibataire et qu'il me serait difficile d'aller au centre-ville en autobus en plein hiver avec mon bébé. Elle m'a dit qu'elle ne pouvait pas appelerla bibliothèque centrale pourmoi. Je l'ai remerciée et suis partie.

Tableau 5. Le modèle de qualité Parasuraman et al. (1985)

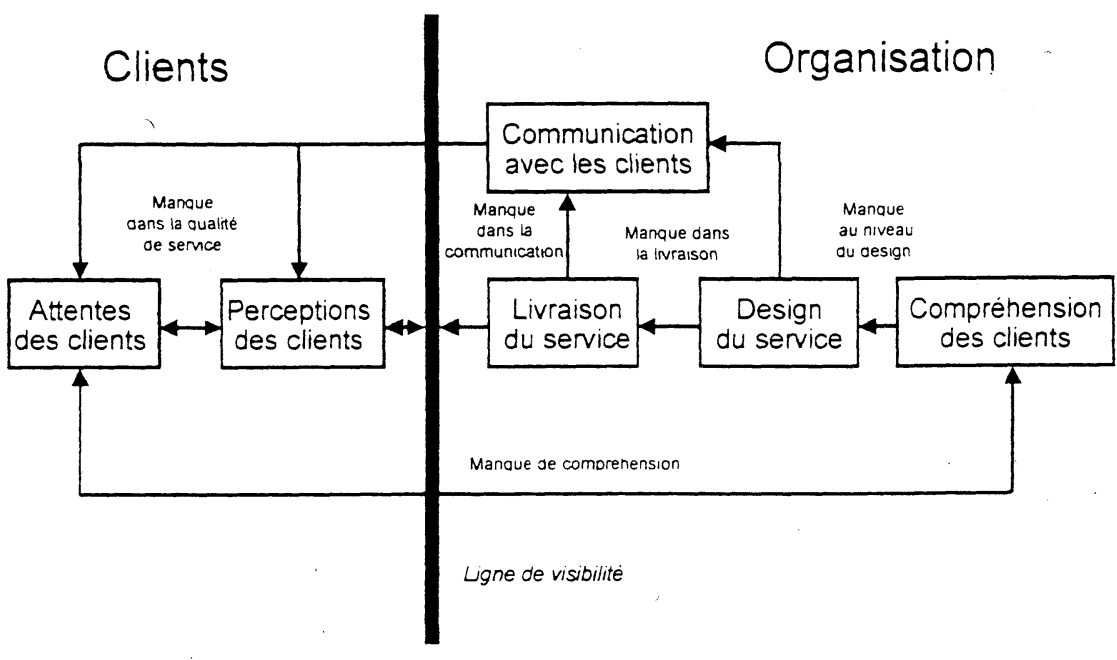

Comment améliorer les services de bibliothèque?

Ces exemples esquissent ce qui s'est passé lorsque 130 participants sont entrés dans les 38 plus importantes bibliothèques publiques du Canada, à la recherche de livres que les bibliothèques ne possédaient pas. Comment améliorer la situation? Parasuraman, Zeithaml and Berry (1990) ont développé un modèle de qualité de service qui inscrit le «quoi» et le "comment» de l'usager dans un modèle organisationnel de qualité (tableau 5).

Ces chercheurs ont découvert cinq moments pendant lesquels des écarts peuvent survenir entre la conception des services et leur livraison à l'usager. Ces écarts représentent les principales difficultés dans les efforts des gestionnaires pour livrer un service que l'usager percevra comme étant de grande qualité.

Écart 1 la différence entre les attentes des usagers et les perceptions de ces attentes parles gestionnaires
Écart 2 la différence entre les perceptions des attentes des usagers par les gestionnaires et les normes ou spécifications déterminées pour le service

Écart 3 la différence entre les normes déterminées pour le service et le service qui est actuellement livré à l'usager

Écart 4 la différence entre le service qui est réellement livré à l'usager et la publicité faite par la bibliothèque des services qu'elle offre

Écart 5 la perspective de l'usager, représentant la différence entre les services attendus et les services perçus. La magnitude et la direction de l'écart 5 sont en conséquence directe des quatre premiers écarts.

Ce modèle offre un cadre de travail qui permet une analyse pratique de la qualité des services et l'introduction d'améliora- 
tions au point précis où elles pourront vite réduire les écarts qui mènent à l'insatisfaction des clients. La réduction de ces écarts aura comme résultat la congruence des évaluations faites par la bibliothèque et par les usagers de la qualité des services de la bibliothèque.

Les bibliothèques ont beaucoup à apprendre sur la manière de donner un bon service. Cela nécessite d'abord une reconnaissance du rôle de la direction d'assurer au personnel de service toutes les ressources, les outils, la formation et la compétence nécessaires pour accomplir un bon travail. Cela requiert également le respect de l'usager et la reconnaissance que la bilbliothèque n'existe que pour répondre aux besoins des usagers. Mais surtout, cela exige une culture de la qualité de service qui doit imprégner toute la bibliothèque où chaque membre du personnel détermine que son travail sera bien fait, la première fois et toutes les fois.

\section{Sources consultées}

Bitner, Mary Jo and Valarie A. Zeithaml. 1987. Fundamentals in service marketing. In Add Value to Your Service: The Key to Success. Edited by Carol Surprenant. Chicago: American Marketing Association, p. 7-11.
Booms, Bernard H. and Jody L. Nyquist. 1981. Analyzing the customer/firm communication component of the services marketing mix. In Marketing of Services. Edited by James $\mathrm{H}$. Donnelly and William R. George. Chicago: American Marketing Association, p. 172-177.

Bowen, David E. and Thomas G. Cummings. 1990. Suppose we took services seriously? In Service Management Effectiveness: Balancing Strategy, Organization and Human Resources, Operations and Marketing. Edited by David E. Bowen et al. San Francisco: Jossey-Bass, p. 1-14.

Davis, Phyllis and Marian Daniel. 1991. Resource sharing and automation: A public library system experience. In Advances in Library Resource Sharing vol. 2. Edited by Jennifer Craghill and Diane J. Graves. Westport, Conn: Meckler, p. 129-134.

Folkes, ValerieS. 1984. Consumer reactions to product failure: An attributional approach. Journal of Consumer Research 10 (March): 398-409.

Grönroos, Christian. 1984. A service quality model and its marketing implications. European Journal of Marketing 18 (4):36-44.

Klaus, Peter G. 1985. Quality epiphenomenon: the conceptual understanding of quality in face-toface service encounters. In The Service Encounter. Managing Employee/Customer Interaction in Service Businesses. Edited by John A. Czepiel, Michael R. Solomon and Carol F. Surprenant. Lexington, Mass: Lexington Books, p. 17-33.

Lancaster, F.W. 1988. If You Want to Evaluate Your Library... Champaign, Ill: University of Illinois Graduate School of Library and Information Science.
Oliver, Richard L. and Wayne S. DeSarbo. 1988. Response determinants in satisfaction judgments. Journal of Consumer Research 14(March): 495507.

Parasuraman, A. 1987. An attributional framework for assessing the perceived value of a service. In Add Value to Your Service: The Key to Success. Edited by Carol Surprenant. Chicago: American Marketing Association, p. 21-24

Parasuraman, A., Valarie A. Zeithaml and Leonard L. Berry. 1988.SERVQUAL: A multiple-item scale for measuring consumer perceptions of service quality. Journal of Retailing 64 (1): 12-40.

Shostack, G. Lynn. 1985. Planning the service encounter. In The Service Encounter: Managing Employee/Customer Interaction in Service Businesses. Edited by John A. Czepiel, Michael R. Solomon, and Carol F. Surprenant. Lexington, Mass: Lexington Books, p. 243-253.

Van House, Nancy A., et al. 1987. Output Measures for Public Libraries: a Manual of Standardized Procedures. Prepared for the Public Library Development Project. 2d ed. Chicago: ALA.

Waldhart, Thomas J. 1985. Performance evaluation of interlibrary loan in the United States: A review of research. Library and Information Science Research 7: 313-331.

Wisconsin Public Library Standards. 1987. Madison: Wisconsin Dept. of Public Instruction.

Zeithaml, Valarie A., A. Parasuraman and Leonard L. Berry. Delivering Quality Service: Balancing Customer Perceptions and Expectations. New York: The Free Press.

\section{PERIODICA}

\section{- PERIODICA Abonnements}

10000 titres (magazines, périodiques et journaux du monde entier)

- PERIODICA Vidéo

1500 titres (arts, sciences, littérature, voyage, jeunesse, cinéma de répertoire)

- BIBLIORAMA

Tous les livres disponibles de langue française distribués au Canada
- BIBLIODATA

Banque de données

des Livres disponibles canadiens de langue française 45000 titres, 516 éditeurs, 250 distributeurs

\section{- Partenaire CEDROM-SNI}

600 banques de données sur CD-ROM

(actualité, affaires, sciences et technologies, santé, médecine, éducation)

Accès direct à plus de 1250 sources

d'information électronique

Demandez nos catalogues : La réponse à vos recherches d'outils pédagogiques de langue française.

PERIODICA INC.

Case postale 444, Outremont

Québec, Canada H2V 4R6
Tél. : (514) 274-5468

Fax : (514) 274-0201

Tout le Canada : 1-800-361-1431 